\title{
Extended Release Granule for Oral Suspension
}

National Cancer Institute

\section{Source}

National Cancer Institute. Extended Release Granule for Oral Suspension. NCI Thesaurus. Code C149873.

Solid preparation consisting of prolonged-release granules intended to be dispersed in the specified liquid to obtain an oral suspension. 